12E getegatine 25) YEARS

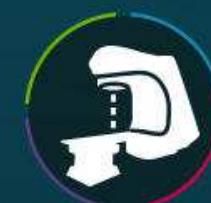

Fully-Automated TG-142 Tests

$\underbrace{}_{\equiv}$

Automated QA for Helical TomoTherapy ${ }^{\circledast}$ TG-148 Tests

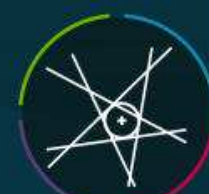

3D Stereotactic Alignment Isocenter Analysis

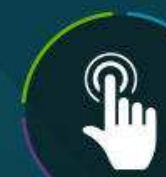

One-Click, Instant Analysis

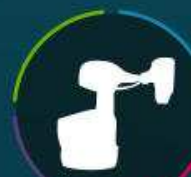

Full CyberKnife ${ }^{\oplus}$ TG-135

Machine QA
THE RIT FAMILY OF PRODUCTS: MEDICAL PHYSICS' LEADING QA SOFTWARE FOR 25 YEARS 


\section{Evaluation of target coverage and margins adequacy during CyberKnife Lung Optimized Treatment}

Running title: target coverage in lung CyberKnife treatments

Keywords: lung stereotactic radiotherapy; CyberKnife; lung optimized treatment; LOT; target coverage; margins.

Rosalinda Ricotti M.Sc. (1) ${ }^{\circ *}$, Matteo Seregni Ph.D. (2) ${ }^{\circ}$, Delia Ciardo M.Sc.(1), Sabrina Vigorito M.Sc.(3), Elena Rondi M.Sc.(3), Gaia Piperno M.D. (1), Annamaria Ferrari M.D. (1), Maria Alessia Zerella MD (1,4), Simona Arculeo M.D. (1,4) Claudia Maria Francia M.D. (1,4), Daniela Sibio M.D. $(1,4)$, Federica Cattani M.Sc. (3), Filippo De Marinis M.D. (5), Lorenzo Spaggiari M.D. Ph.D (4,5), Roberto Orecchia M.D. (4,6), Marco Riboldi Ph.D. (2), Guido Baroni Ph.D.(2,7) § Barbara Alicja Jereczek-Fossa M.D., Ph.D.(1,4) §

1 Division of Radiation Oncology, European Institute of Oncology, Milan - Italy

2 Dipartimento di Elettronica Informazione e Bioingegneria, Politecnico di Milano, Milan - Italy

3 Unit of Medical Physics, European Institute of Oncology, Milan - Italy

4 Department of Oncology and Hemato-oncology, University of Milan, Milan - Italy

5 Division of Thoracic Surgery, European Institute of Oncology, Milan - Italy

6 Department of Medical Imaging and Radiation Sciences, European Institute of Oncology, Milan Italy

7 Bioengineering Unit, Centro Nazionale di Adroterapia Oncologica (CNAO), Pavia - Italy

- Rosalinda Ricotti and Matteo Seregni equally contributed to this work and should be considered as co-first authors

$\S$ Guido Baroni and Barbara Alicja Jereczek-Fossa should be considered as co-last authors

* Corresponding author

Ricotti Rosalinda, M.Sc.

Division of Radiation Oncology, European Institute of Oncology

via Ripamonti 435 (20141), Milan, Italy.

Phone number:+390294372615

This article has been accepted for publication and undergone full peer review but has not been through the copyediting, typesetting, pagination and proofreading process, which may lead to differences between this version and the Version of Record. Please cite this article as doi: $10.1002 / \mathrm{mp} .12804$

This article is protected by copyright. All rights reserved. 
E-mail: rosalinda.ricotti@ieo.it.

rosalinda.ricotti@gmail.com.

\begin{abstract}
Purpose

Evaluation of target coverage and verification of safety margins, in motion management strategies implemented by Lung Optimezed Treatment (LOT) module in CyberKnife system.
\end{abstract}

\title{
Methods
}

Three fiducial-less motion management strategies provided by LOT can be selected according to tumour visibility in the X-ray images acquired during treatment. In 2-view modality the tumor is visible in both X-ray images and full motion tracking is performed. In 1-view modality the tumor is visible in a single X-ray image, therefore motion tracking is combined with an internal target volume (ITV)-based margin expansion. In 0-view modality the lesion is not visible, consequently the treatment relies entirely on an ITV-based approach.

Data from 30 patients treated in 2-view modality were selected providing information on the threedimensional tumor motion in correspondence to each X-ray image. Treatments in 1-view and 0-view modalities were simulated by processing log files and planning volumes. Planning target volume (PTV) margins were defined according to the tracking modality: end-exhale clinical target volume (CTV) $+3 \mathrm{~mm}$ in 2-view and ITV $+5 \mathrm{~mm}$ in 0-view. In the 1-view scenario, the ITV encompasses only tumor motion along the non-visible direction. Then, non-uniform ITV to PTV margins were applied: 3 $\mathrm{mm}$ and $5 \mathrm{~mm}$ in the visible and non-visible direction, respectively.

We defined the coverage of each voxel of the CTV as the percentage of X-ray images where such voxel was included in the PTV. In 2-view modality coverage was calculated as the intersection between the CTV centred on the imaged target position and the PTV centred on the predicted target position, as recorded in log files. In 1-view modality, coverage was calculated as the intersection between the CTV centred on the imaged target position and the PTV centred on the projected

This article is protected by copyright. All rights reserved. 
predictor data. In 0-view modality coverage was calculated as the intersection between the CTV centred on the imaged target position and the non-moving PTV.

Similarly to dose-volume histogram, CTV coverage-volume histograms (defined as $\mathrm{CVH}$ ) were derived for each patient and treatment modality. The geometric coverages of the $90 \%$ and $95 \%$ of CTV

volume (C90, C95 respectively) were evaluated. Patient-specific optimal margins (ensuring C95 $\geq 95 \%$ ) were computed retrospectively.

\section{Results}

The median \pm interquartile-rage of C90 and C95 for upper lobe lesions was $99.1 \pm 0.6 \%$ and $99.0 \pm$ $3.1 \%$, whereas they were $98.9 \pm 4.2 \%$ and $97.8 \pm 7.5 \%$ for lower and middle lobe tumours.

In 2-view, 1-view and 0-view modality, adopted margins ensured c95 $\geq 95 \%$ in $70 \%, 85 \%$ and $63 \%$ of cases and C95 $\geq 90 \%$ in $90 \%, 88 \%$ and $83 \%$ of cases respectively. In 2-view, 1-view and 0-view a reduction of margins still ensured C95 $\geq 95 \%$ in 33\%, $78 \%$ and $59 \%$ of cases respectively.

\section{Conclusions}

CTV coverage analysis provided an a-posteriori evaluation of the treatment geometric accuracy and allowed a quantitative verification of the adequacy of the PTV margins applied in CyberKnife LOT treatments offering guidance in the selection of CTV margins.

\section{Introduction}

Stereotactic body radiotherapy (SBRT) is the preferred treatment option for inoperable lung tumours, given its limited treatment-related toxicity and its good local control rates [1-4]. Moreover, it is recently considered as a viable treatment option also for operable lung patients [5]. However, lung tumours are prone to motion (mainly caused by respiration) that affects both intrafractional and interfractional radiation delivery [6]. The management of respiratory motion in lung SBRT is essential to achieve high therapeutic ratio, ensuring adequate coverage of the moving target volume

This article is protected by copyright. All rights reserved. 
while sparing the surrounding critical structures. The amount of irradiated volume depends on the applied motion management strategy and on the adopted safety margins [7].

The main aim of the present study was providing a quantitative evaluation of target coverage, as a function of planning target volume margins, in the three fiducial-less motion management strategies implemented by Lung Optimezed Treatment (LOT) module in CyberKnife ${ }^{\circledR}$ system (Accuray, Inc., Sunnyvale, CA). We pursued such aim through retrospective analysis of pre-treatment information derived from 4DCT-based planning and in-treatment data extracted from log files.

\section{Materials and Methods}

\section{Lung Optimized Treatment (LOT) module of CyberKnife system}

Recently, CyberKnife ${ }^{\circledR}$ system (Accuray, Inc., Sunnyvale, CA) has been equipped with Lung Optimized Treatment (LOT) module (Fig. 1). It provides three fiducial-less motion management strategies, to be selected according to tumour visibility in the X-ray images acquired during treatment: (i) dynamic tumour tracking (2-view modality), (ii) internal target volume (ITV)-based approach (0-view modality) and (iii) combined tracking and ITV-based approach (1-view modality).

In 2-view modality, the tumour is localized in both orthogonal X-ray images and three dimensional (3D) motion tracking is performed as in Xsight Lung Tracking ${ }^{\mathrm{TM}}[8,9]$. In 0-view modality, the tumour cannot be detected in either of the X-ray images and consequently the treatment relies entirely on an ITV-based approach, using Xsight Spine tracking ${ }^{\mathrm{TM}}$ for patient alignment $[10,11]$. The irradiated volume encompasses tumour motion, as detected by pre-treatment four-dimensional computed tomography (4D-CT). In 1-view modality, the tumour is visible in only one of the X-ray projections (A or B) and dynamic tumour tracking compensates the target motion only in the detectable plane. Non-visible motion is compensated with an ITV-based strategy. Therefore, less normal tissue is exposed to radiation compared to the ITV-based approach. Usually, the visibility of the target in the X-ray images, and thus the selection of motion management modalities, depends on target size,

This article is protected by copyright. All rights reserved. 
density and location $[10,8,12]$. At our Institution about $55 \%$ of lung patients undergoing CyberKnife SBRT are treated in 0-view modality, $27 \%$ in 1-view modality and $18 \%$ in 2-view-modality [13].

\section{Patient data}

This study included data from 30 patients, treated for lung tumours with CyberKnife in 2-view modality from November 2014 to February 2017. The study was performed within the Institutional Ethics Committee notification regarding stereotactic body radiotherapy and image-guided radiotherapy (notifications $n^{\circ} 93 / 11$ ). The patients gave written informed consent for radiotherapy and for the use of their anonymized data for educational and research purposes.

At the time of radiation treatment, the median patient age was 76 years (range: $46-87$ years). Thirteen patients reported respiratory disorders, mainly chronic obstructive pulmonary disease. Planning 4D-CT was acquired by GE Optima CT580 W scanner (GE Healthcare, Chicago, IL, US) featuring $1.25 \mathrm{~mm}$ slice thickness and $1.27 \mathrm{~mm}$ pixel spacing. The Real-time Position Management system (RPM, Varian, Palo Alto, US) was used for amplitude-based sorting. Full-inhale and full-exhale phases were considered for treatment planning. At our Institution, the planning target volume (PTV) in 2-view modality was defined as $3 \mathrm{~mm}$ isotropic expansion of the exhale clinical target volume (CTV). We choose the exhale phase as a reference state as it is the most reproducible and representative phase of free breathing since tumor spent more time in the exhalation than in the inhalation phase [14]. All patients were positioned using a customized external vacuum-type cast. The size of the CyberKnife collimator varied between 25- $60 \mathrm{~mm}$ and was comparable to the size of the lesion ensuring the PTV irradiation along its entire movement. Treatment consisted of 3-5 fractions to a total dose ranging from 18 to $54 \mathrm{~Gy}$, prescribed to the isodose of $80 \%$ of prescription dose.

Data including the 3D coordinates of the target over the course of treatment were extracted from the treatment log files and used to determinate the actual target motion in the three anatomical directions (latero-lateral (LL), anterior-posterior (AP) or superior inferior (SI)). [6, 12, 15-21].

This article is protected by copyright. All rights reserved. 
Multiplan (version 5.2 Accuray) was used for treatment planning and data analysis was performed with MATLAB $^{\circledR}$ (MathWorks, Natick, MA) and Plastimatch [22].

\section{Tumor motion evaluation}

The amplitude of respiratory motion was measured in 4D-CT data as the distance between the centre of mass of the CTV in the exhale and inhale positions. During treatment, peak-to-peak respiratory amplitude was derived from the tumour positions estimated by the correlation model, according to the method proposed by Lu et al. [23].

The Kruskal-Wallis and Wilcoxon rank sum tests at $1 \%$ significance level investigated differences among motion amplitudes in different anatomical directions, as well as the correspondence between pre-treatment motion amplitude and the median motion amplitude during treatment. Dependence of respiratory motion amplitudes on tumour location and CTV volume was evaluated using Spearman correlation coefficient.

\section{Correlation and overall tracking error}

The correlation error was defined as the discrepancy between the target position estimated by the correlation model and the position measured by the X-ray images during treatment delivery. The overall tracking error considered the predicted target position, thus including both correlation and temporal prediction uncertainties. The dependency of such errors on target motion amplitude in each anatomical direction was investigated through Spearman correlation coefficient.

\section{Coverage evaluation}

CTV coverage was investigated in order to provide a quantification of the treatment geometric accuracy. It was calculated in correspondence to each control image acquired during irradiation as the intersection between the CTV centred on the imaged target position (serving as ground-truth) and the PTV centred on the predicted target position, as recorded in log files. In this way, we define the coverage of each voxel of the CTV as the percentage of control images where such voxel was included in the irradiated volume (i.e. PTV), as exemplified in Figure 2A and 2B.

This article is protected by copyright. All rights reserved. 
The coverage analysis was carried out for the 2-view modality as described above and for simulated 1-view and 0-view modalities. In order to simulate 1-view and 0-view treatment delivery modalities we took advantage of the 2-view tracking data extracted from the treatment log files providing 3D coordinates of the target over the course of treatment . CTV coverage in 1-view and 0-view was calculated as described hereafter.

In the 1-view case, tracking is restricted on the plane perpendicular to the active camera's axis (inplane directions). Consequently, the ITV-1-view was defined as the envelope of the CTV volume at inhale and exhale positions along the camera axis, i.e. encompassing only the out-of-plane target motion, which cannot be imaged since it is parallel to the camera axis (out-of-plane direction). In this scenario, the PTV was obtained, according to the protocol of our Institution, through an anisotropic margin applied to the ITV-1-view: $3 \mathrm{~mm}$ in-plane and $5 \mathrm{~mm}$ in out-of-plane directions. To simulate planar tracking, predicted target locations extracted form log files were projected on in-plane directions. Then, coverage was calculated as the intersection between the CTV centred on the imaged target position and the PTV centred on the projected predictor data.

In 0-view treatments, tracking is disabled. Therefore, the ITV was defined as the envelope of the CTV at the inhale and the exhale phase of the planning 4D CT. Then, the PTV was derived by $5 \mathrm{~mm}$ isotropic margin expansion from the ITV. In this case, we computed CTV coverage as the intersection between the CTV centred on the imaged target position and the non-moving PTV.

Similarly to cumulative dose-volume histograms, coverage-volume histograms (CVH) were derived from volumetric coverage distributions. The coverage of the $90 \%$ and the $95 \%$ of CTV volume (C90 and C95) were chosen as a CVH evaluation metrics (Figure 2C). CVH, C90 and C95 values were derived for each patient and for the three LOT modalities: 2-view, 1-view (simulating both 1-View-A and 1-View-B scenarios depending on the active camera) and 0-view.

We considered the coverage of $95 \%$ of CTV volume greater than $95 \%$ (C95 $\geq 95 \%$ ) as a threshold to

This article is protected by copyright. All rights reserved. 
determine whether the applied margin ensured adequate coverage of the target volume $[7,12,17]$.

The Kruskal-Wallis test was applied to C90 and C95 distributions to compare target coverage obtained with the different modalities. Furthermore, the influence of tumour position on coverage was investigated with the Wilcoxon rank sum test.

\section{Margin optimization}

We adopted the above-mentioned margin evaluation criterion (C95 $\geq 95 \%$ ) to apply an a-posteriori margin optimization on a patient-specific basis.

In the 2-view modality, the CTV volume was gradually expanded by adding $1 \mathrm{~mm}$ isotropic margins until reaching $\mathrm{C} 95 \geq 95 \%$. The 0 -view modality was treated in the same way, but the incremental margins were applied to the ITV.

For the 1-view modality, in accordance to the current clinical protocol, we adopted an anisotropic margin optimization approach: the ITV-1view (as defined in the previous paragraph) was progressively expanded evaluating each combination of in-plane and out-of-plane margin between 2 and $12 \mathrm{~mm}$, then choosing the combination that satisfies the $\mathrm{C} 95 \geq 95 \%$ criterion minimizing the final volume of the PTV.

For each patient, the optimal margin was computed for each modality and the resulting volume of PTV volume was compared against the CTV volume to provide a synthetic measurement of the expansion required to obtain adequate coverage.

\section{Results}

A total of 96 treatment fractions corresponding to an average total treatment time over fractions per patients of 64 minutes (range: $31-156$ minutes, from the first to the last treatment node) were analysed. X-ray images were acquired periodically during treatment with a median time interval of 57 s (range: $21-93$ s).

This article is protected by copyright. All rights reserved. 
In 19, 2 and 9 patients, tumours were located in the upper, middle and lower lobes, respectively. Fourteen tumours were left-sided while sixteen were right sided. Median \pm interquartile range (IQR) of tumour distance from the spine was $76.9 \pm 23.8 \mathrm{~mm}$ (range: $44.0-138.6 \mathrm{~mm}$ ). Median \pm IQR of CTV volume in the exhale phase was $19.6 \pm 32.3 \mathrm{~cm}^{3}$ (range: $1.1-112.0 \mathrm{~cm}^{3}$ ).

\section{Tumor motion evaluation}

For all patients, the respiratory motion measured in the 4D-CT was $0.6 \pm 1.0 \mathrm{~mm}$ (median \pm IQR), 1.2 $\pm 1.7 \mathrm{~mm}$ and $4.2 \pm 6.0 \mathrm{~mm}$ in LL, AP and SI direction, respectively. Respiratory amplitude during treatment was $0.9 \pm 1.0 \mathrm{~mm}, 1.2 \pm 1.6 \mathrm{~mm}$ and $4.6 \pm 8.3 \mathrm{~mm}$ in LL, AP and SI direction, respectively. The predominant direction of tumour motion was SI both in the 4D-CT analysis and in treatment log files analysis $(p<<0.01)$.

Median SI motion amplitude was significantly larger in lower/middle lobes compared to the upper lobe both in 4D-CT scenario and during treatment $(p<<0.01)$. Motion amplitude was not significantly different $(p>0.09)$ between 4D-CT scenario and during treatment.

No dependence of median motion amplitude on CTV volume was found $(R<0.5, p>0.01)$ neither in 4D-CT study nor during treatment. Similarly, no dependence on the distance from the spine was found $(R<0.5, p>0.01)$.

\section{Correlation and overall tracking error}

The median \pm IQR of the correlation error of all patients was $0.5 \pm 0.7\left(95^{\text {th }}\right.$ percentile: 1.9$) \mathrm{mm}, 0.6 \pm$ 0.9 (2.7) $\mathrm{mm}, 0.7 \pm 1.1(3.6) \mathrm{mm}$ in LL, AP and SI direction, respectively. Overall tracking errors were almost identical to correlation errors: $0.5 \pm 0.7(1.9) \mathrm{mm}$ in $\mathrm{LL}$ direction, $0.6 \pm 0.9(2.7) \mathrm{mm}$ in AP and $0.8 \pm 1.3(3.7) \mathrm{mm}$ in SI.

Correlation was found between the correlation error and tumour motion amplitudes captured during irradiation in $\mathrm{AP}(\mathrm{R}=0.57, p=0.001)$ and $\mathrm{SI}(\mathrm{R}=0.81, p<<0.01)$ directions. Similarly, correlation was found between overall tracking error and amplitudes during treatment in $\operatorname{AP}(R=0.54, p=0.002)$ and $\mathrm{SI}(\mathrm{R}=0.84, p=<<0.01)$ directions.

This article is protected by copyright. All rights reserved. 


\section{Coverage evaluation}

The CVHs obtained for each LOT modality and each patient are shown in Figure 3 while the CVH evaluation metrics (C90 and C95) are summarized in Table 1 and extensively shown in Supplementary Table 1.

Even if median C95 and C90 were slightly smaller for 2-view treatments with respect to 1-view and 0-view, no statistically significant difference were observed among the C90 and C95 population corresponding to the three modalities ( $p=0.42$ and $p=0.52$ respectively). Target coverage was equally guaranteed in the different treatment modalities.

Considering the treatment modalities altogether, we observed that C90 and C95 depended on the tumour position in the lung. The Wilcoxon rank sum test showed significant difference between the two populations corresponding to upper lobe and lower/middle lobe $(p<<0.001$ for both $C 90$ and C95). The median \pm IQR of C90 for upper lobe lesions was $99.1 \pm 0.6 \%$ (range: $55.0-99.1 \%$ ), whereas it was $98.9 \pm 4.2 \%$ (range: $73.1-99.1 \%$ ) for lower and middle lobe tumours. Similarly, the median \pm IQR of C95 for upper lobe lesions was $99.0 \pm 3.1 \%$ (range: $52.9-99.1 \%$ ), whereas it was $97.8 \pm 7.5 \%$ (range: $60.9-99.1 \%$ ) for lower and middle lobe tumors.

In 2-view scenario, the current prescription of $3 \mathrm{~mm}$ isotropic margins expansion around the CTV in end-exhale ensured $195 \geq 95 \%$ in $21 / 30$ (70\%) patients. Among the 9 patients who did not meet the criterion of $c 95 \geq 95 \%$, only 3 patients had $C 95 \leq 90 \%$. In these patients, tumours were located in the middle and lower lobes and motion excursion measured in the 4D-CT were greater than the median motion excursion of the population. These patients exhibited $C 95 \leq 90 \%$ also in the other treatment modalities.

In Figure 4, the CTV coverage in 2-view modality is reported for the patient showing the worst C95 within the population ( $C 95=77.2 \%$ ).

This article is protected by copyright. All rights reserved. 
In the simulated 1-view scenario, in-plane expansion of $3 \mathrm{~mm}$ and out-of-plane expansion of $5 \mathrm{~mm}$ ensured adequate CTV coverage (C95 $\geq 95 \%$ ) in 51/60 (85\%) of 1-view cases (A-B). In particular, 5 patients exhibited $c 95 \leq 90 \%$.

Finally, in the simulated 0-view scenario, $5 \mathrm{~mm}$ isotropic margin expansion around the ITV ensured adequate CTV coverage in 19 (63\%) patients. Among the 9 patients who did not meet the criterion of C95 $\geq 95 \%, 5$ patients exhibited C90 and C95 $\leq 90 \%$. This was probably due to prolonged irregularities in the breathing pattern during irradiation).

\section{Margin optimization}

Margin optimization results are reported in Figure 5.

In 2-view scenario, margin expansion of the CTV could be reduced to $2 \mathrm{~mm}$, still ensuring C95 $\geq 95 \%$ in $10(33 \%)$ patients. However, in $9(30 \%)$ patients, a larger margin (up to $7 \mathrm{~mm}$ ) was required to meet the above-mentioned criterion.

Considering the simulated 1-view scenario, $3 \mathrm{~mm}$ margin expansion in in-plane and out-of-plane directions allowed to achieve the desired converge in $71.7 \%$ of the cases (considering 1-view- $A$ and 1-view-B altogether). Nevertheless, in a single instance a very large margin (isotropic $10 \mathrm{~mm}$ ) was required.

Finally, in the simulated 0-view scenario, in 18 (60\%) patients, margin expansion could be reduced still ensuring target coverage. However, in $11(37 \%)$ patients larger margins were necessary to ensure at least $95 \%$ of coverage to the $95 \%$ of CTV volume.

The Kruskal-Wallis test did not report any significant difference between volumes of PTV defined according to the current prescriptions and those obtained through patient-specific margin optimization for each treatment modalities ( $p>0.7)$. In both 2-view and 1-view modality, optimal margin resulted in slightly larger PTV volume, whereas the opposite behaviour was observed in 0view cases.

This article is protected by copyright. All rights reserved. 


\section{Discussion}

In this work, we considered data from 30 patients treated with CyberKnife LOT. Through the quantification of the CTV geometric coverage, we investigated the adequacy of the PTV margins applied in our Institution simulating different treatment modalities (2-view, 1-view and 0-view). Results showed that such margins ensure the required target coverage in the majority of the cases.

A preliminary analysis of tumor motion was performed. The respiratory amplitude measured with planning 4D-CT and with online tumour tracking data was in agreement with previously published studies $[12,6,14,23,24]$. In addition, motion amplitudes captured with 4D-CT were representative of median excursions measured during treatment for each anatomical direction and in each lung lobe $[25,26,23]$. This confirms that the ITV, defined during treatment planning as the envelope of exhale and inhale CTV, is a robust approximation of tumour motion observed during irradiation, despite inter- and intra-fractional variations.

Several retrospective studies calculated the uncertainty in radiation delivery (i.e. tumour tracking errors) by analysing dynamic tracking log files produced by the CyberKnife system [12,15-20, 28, 29]. Our results were in agreement with these works: limited tracking errors (median $<1 \mathrm{~mm}$ ) were observed in all directions and a significant correlation was found between the errors magnitude and motion amplitude.

We relied on CTV coverage as a metric to quantify geometric treatment accuracy. In this way, tracking errors (in 2-view and 1-view modality along the visible directions) and non-tracked tumour motion (in 1-view modality along the non-visible direction and in 0-view) can be considered altogether and are effectively related to the adopted margins., Moreover, geometric tumour coverage is an essential requirement for dose coverage and our analysis could represent a preliminary examination for further investigation on dose distribution actually delivered to the CTV throughout the entire treatment taking into account the temporal interplay between the CTV motion inside the PTV and the temporal aspect of the actual dose delivery.

This article is protected by copyright. All rights reserved. 
The safety margins adopted in our Institution ensured a median C95 greater that 95\% in each LOT modality. This means that, considering the entire patient population, the margins met the coverage criterion of C95 $\geq 95 \%$. Consequently, patient-specific margin optimization resulted in larger margins in less than $37 \%$ of patients considering all LOT modalities. In 2-view modality, larger margins are required in $30 \%$ of patients to compensate mostly for tracking errors. Indeed, in 2-view, C95 is strongly correlated with the overall tracking error $(R=-0.9)$. Conversely, in 0 -view modality $C 95$ is strongly correlated with tumour motion measured during treatment $(R=-0.8)$. Actually, 0 -view modality is more sensitive to interfraction and intrafraction breathing pattern variations, which are likely to occur in patients with respiratory disease. Similarly to our work, Descovitch et al. [12] evaluated target coverage simulating 0 -view modality treatments and concluded that a uniform margin of $4.5 \mathrm{~mm}$ around the ITV was necessary to assure $95 \%$ target coverage for $95 \%$ of the fractions. With respect to Descovich et al, we further analysed target coverage in 1- and 2-view modality. In 1-view, C95, and consequently the optimal margin, depends on both tracking errors ( $\mathrm{R}=-$ $0.7)$ and respiratory motion $(\mathrm{R}=-0.6)$. However, in some patients, safety margins could be reduced still ensuring CTV coverage, while enhancing treatment conformality by sparing a larger volume of healthy tissues.

Irradiated volumes increases passing from 2-view to 1-view and 0-view modalities. Considering the same CTV coverage (C95 $\geq 95 \%$ ), the use of 1-view of 0-view modalities instead of 2-view resulted in PTV enlarged by $36.6 \pm 25.8 \%$ and $82.9 \pm 101.2 \%$, respectively.

The visibility of the target in the x-ray images acquired during treatment (and thus the selection of the suitable motion management modality) depends on target size, density and location. The patients included in this study were treated with 2-view tracking modality; hence all lesions had characteristics that made them visible in both X-ray images. The presented coverage analysis was then extended to simulated 1-view and 0-view modalities taking advantage of the complete 2-view tracking data extracted from the treatment log files. In clinical practice, the 1-view and 0-view

This article is protected by copyright. All rights reserved. 
modalities are selected when the lesions are too small, not sufficiently dense or when they overlap organs at risk (OAR) in the x-ray projections. This could occur regardless of the type of patient's breathing motion. This means that the same lesion, characterized by the same movement during breathing, could turn out to be a 2-view, 1-view and 0-view case at varying its density and size resulting visible or not in the x-ray images acquired during treatment. From this point of view, the coverage analysis performed in simulated 1-view and 0-view cases could be considered realistic. However, in 0-view treatments tissue shifts between spine and lesion could occur and their effect on CTV coverage cannot be considered in our analysis and specific further investigation are advisable.

In conclusion, safety margins allow compensating tracking errors and tumor motion in all LOT modalities ensuring adequate target coverage in the majority of patients. CTV coverage analysis provided an a-posteriori evaluation of the treatment geometric accuracy and allowed us to quantitatively verify the adequacy of the PTV margins applied in CyberKnife LOT treatments delivered at our Institution. Further investigations on dose distribution actually delivered to the CTV throughout the entire treatment will be addressed to fully evaluate the adequacy of safety margins.

\section{Figure Legends}

Figure 1: Exemplification of planning target volumes (PTVs) in different Lung Optimized Treatment (LOT) modalities as simulated on a 2-view patient. Axial, sagittal and coronal slices are shown in panels $A, B$ and $C$ respectively. Yellow, green, magenta and red contours refer to PTVs corresponding to 2-view, 1-view-A, 1view-B and 0-view modality, respectively.

Figure 2: Exemplification of the CTV coverage calculation. Panel A: for each control image acquired during treatment $(1,2, \ldots, N)$ the intersection between the CTV (green circle) and the PTV (red circle) is calculated and accumulated. Panel B: the coverage of a given voxel within the CTV expresses the percentage of control images where the PTV covers that voxel. Panel C: cumulative coverage-volume histogram (CVH). In this exemplification, about $17 \%$ of the volume receives $100 \%$ coverage, whereas $62 \%$ of the volume receives at least $66 \%$ coverage and $100 \%$ of the volume has a coverage equal or larger than $33 \%$. C95 is 33\%, meaning that $95 \%$ of the volume shows a coverage of at least $33 \%$.

Figure 3: Converge-volume histograms $(\mathrm{CVH})$. Blue lines show $\mathrm{CVH}$ of individual patients, the patient-wise median is represented in red. Panels $A, B$ and $C$ refer to 2-view, 1-view and 0-view scenarios, respectively. In panel B, $60 \mathrm{CHV}$ are reported, since each patient was simulated in both 1-view-A and 1-view-B cases. Panels C,

This article is protected by copyright. All rights reserved. 
$\mathrm{E}$ and $\mathrm{F}$ show an expanded view of the $80 \%$ to $100 \%$ box in the upper-right corner of the $\mathrm{CVH}$. Abbreviations: $\mathrm{CTV}=$ clinical target volume, $\mathrm{CVH}=$ coverage-volume histogram.

Figure 4: Example of coverage distribution within the clinical target volume (CTV). Panel A: rendering of the patients anatomy, the red surface shows the planning target volume (PTV) in the right lower lobe. Axial and coronal slices intersecting in tumor center are also reported. Panel B: CTV coverage on the central axial slice color-coded as reported in the color bar. Magenta, red and green contours represent CTV, clinical PTV (CTV + $3 \mathrm{~mm}$ ) and optimal PTV ensuring C95 $\geq 95 \%$ (CTV $+7 \mathrm{~mm})$, respectively. Panel C: central coronal slice.

Figure 5: Margin optimization results. Panel A: optimal margins for 2-view treatments. The bar plot shows the frequency of a given optimal margin. Panel B: 1-view scenario. Each bar of the matrix corresponds to a combination of in-plane and out-of-plane margins. Frequency is color-coded from blue to yellow. Panel C: 0View scenario, represented as in panel A. In all panels, the margin prescription corresponding to the current clinical protocol is highlighted with a red box on the axes.

Acknowledgment: This work was partially supported by a research grant from Accuray Inc. entitled "Data collection and analysis of Tomotherapy and CyberKnife breast clinical studies, breast physics studies and prostate study". The Sponsors did not play any role in the study design, collection, analysis and interpretation of data, nor in the writing of the manuscript, nor in the decision to submit the manuscript for publication.

Disclosure of Conflict of Interest: The authors have no conflicts of interest to disclose

This article is protected by copyright. All rights reserved. 


\section{References}

[1] Timmerman R, Paulus R, Galvin J, et al. Stereotactic body radiation therapy for inoperable early stage lung cancer, JAMA. 2010 Mar 17;303(11):1070-6. doi: 10.1001/jama.2010.261.

[2] Senthi S, Lagerwaard FJ, Haasbeek C J A, Slotman BJ, Senan S. Patterns of disease recurrence after stereotactic ablative radiotherapy for early stage non-smallcell lung cancer: a retrospective analysis, Lancet Oncol. 13 (8) (2012) 802-809. doi: 10.1016/S1470-2045(12)70242-5.

[3] Nuyttens JJ, van der Voort van Zyp NC, Praag J, et al. Outcome of four-dimensional stereotactic radiotherapy for centrally located lung tumors. Radiother Oncol. 2012 Mar;102(3):383-7. doi: 10.1016/j.radonc.2011.12.023.

[4] van der Voort van Zyp NC, Prévost JB, Hoogeman MS, et al. Stereotactic radiotherapy with realtime tumor tracking for non-small cell lung cancer: clinical outcome. Radiother Oncol. 2009 Jun;91(3):296-300. doi: 10.1016/j.radonc.2009.02.011

[5] Chang JY, Senan S, Paul MA, et al. Stereotactic ablative radiotherapy versus lobectomy for operable stage I non-small-cell lung cancer: a pooled analysis of two randomised trials. Lancet Oncol 2015; 16: 630-37. doi: https://doi.org/10.1016/S1470-2045(15)70168-3

[6] Knybel L, Cvek J, Molenda L, et al. Analysis of lung tumor motion in a large sample: patterns and factors influencing precise delineation of internal target volume. Int $\mathrm{J}$ Radiat Oncol Biol Phys. 2016 Nov 15;96(4):751-758. doi: 10.1016/j.ijrobp.2016.08.008.

[7] International Commission on Radiation Units and Measurements ICRU Report 62. Prescribing, recording, and reporting photon beam therapy (Supplement to ICRU Report 50), ICRU, Bethesda, MD (1999)

[8] Bahig H, Campeau MP, Vu T, et al. Predictive parameters of CyberKnife fiducial-less (XSight Lung) applicability for treatment of early non-small cell lung cancer: A single-center experience. Int J Radiat Oncol Biol Phys. 2013 Nov 1;87(3):583-9. doi: 10.1016/j.jijobp.2013.06.2048.

[9] Fu D, Kahn R, Wang B, et al. Xsight Lung Tracking System: A Fiducial-Less Method for Respiratory Motion Tracking In Robotic Radiosurgery. Treating Tumors that Move with Respiration, 1, 2007;265.

[10] Bondiau P. Y. Cyberknife tracking methods In The Revolution of Robotic Stereotactic Radiotherapy (Robotic Radiosurgery) in Lung Cancer, 2014; 93-100.

[11] Guo Y, Zhuang H, Zhao L, Yuan Z, Wang P. Influence of different image-guided tracking methods upon the local efficacy of CyberKnife treatment in lung tumors. Thorac Cancer. 2015 May;6(3):255-9. doi: 10.1111/1759-7714.12174.

[12] Descovich M, McGuinness C, Kannarunimit D, et al. Comparison between target margins derived from 4DCT scans and real-time tumor motion tracking: Insights from lung tumor patients treated with robotic radiosurgery. Med Phys. 2015 Mar;42(3):1280-7. doi: 10.1118/1.4907956.

[13] Sibio D, Francia CM, Zerella MA. et al. Retrospective evaluation of Cyberknife ${ }^{\circledR}$ in the treatment of lung lesions: clinical outcome and toxicity profile. Proceedings of Italian Association of Radiation Oncology (AIRO) congress 2016 available at https://www.radioterapiaitalia.it/wpcontent/uploads/2017/06/AIRO-2016.pdf p. 102

[14] Seppenwoolde, Y, Shirato H, Kitamura K, et al. Precise and real-time measurement of 3D tumor motion in lung due to breathing and heartbeat, measured during radiotherapy. Int $\mathrm{J}$ Radiat Oncol Biol Phys. 2002 Jul 15;53(4):822-34

[15] Yang ZY, Chang Y, Liu HY, Liu G, Li Q. Target margin design for real-time lung tumor tracking stereotactic body radiation therapy using CyberKnife Xsight Lung Tracking System. Sci Rep. 2017 Sep 7;7(1):10826. doi: 10.1038/s41598-017-11128-w.

[16] Floriano A, García R, Moreno R et al. Retrospective evaluation of CTV to PTV margins using CyberKnife in patients with thoracic tumors. J Appl Clin Med Phys. 2014 Nov 8;15(6):4825. doi: 10.1120/jacmp.v15i6.4825.

[17] Pepin E W, Wu H, Zhang $\mathrm{Y}$ et al. Correlation and prediction uncertainties in the cyberknife synchrony respiratory tracking system. Med Phys. 2011 Jul;38(7):4036-44. doi:10.1118/1.3596527

[18] Hoogeman M, Prévost JB, Nuyttens $\mathrm{J}$ et al.. Clinical accuracy of the respiratory tumor tracking system of the cyberknife: assessment by analysis of log files. Int J Radiat Oncol Biol Phys. 2009 May 1;74(1):297-303. doi: 10.1016/j.jrobp.2008.12.041.

[19] Malinowski KT, McAvoy TJ, George R, Dieterich S, D'Souza WD. Mitigating errors in external

This article is protected by copyright. All rights reserved. 
respiratory surrogate-based models of tumor position. Int J Radiat Oncol Biol Phys. 2012 Apr 1;82(5):e709-16. doi: 10.1016/j.jirobp.2011.05.042.

[20] Winter JD, Wong R, Swaminath A, Chow T. Accuracy of Robotic Radiosurgical Liver Treatment Throughout the Respiratory Cycle. Int J Radiat Oncol Biol Phys. 2015 Nov 15;93(4):916-24. doi: 10.1016/j.jijrobp.2015.08.031.

[21] Seppenwoolde Y, Berbeco RI, Nishioka S, Shirato H, Heijmen B. Accuracy of tumor motion compensation algorithm from a robotic respiratory tracking system: a simulation study. Med Phys. 2007 Jul;34(7):2774-84

[22] Sharp G, Li R, Wolfgang J, Chen G, Peroni M, Spadea MF, Mori S, Zhang J, Shackleford J, Kandasamy N. Plastimatch-An open source software suite for radiotherapy image processing. In Proceedings of the XVI'th International Conference on the use of Computers in Radiotherapy (ICCR), Amsterdam, Netherlands 2010 May.

[23] Lu W, Nystrom MM, Parikh PJ, et al. A semi-automatic method for peak and valley detection in free-breathing respiratory waveforms. Med Phys. 2006 Oct; 33(10):3634-6. doi: 10.1118/1.2348764

[24] Harada K, Katoh N, Suzuki R, et al. Evaluation of the motion of lung tumors during stereotactic body radiation therapy (SBRT) with four-dimensional computed tomography (4DCT) using realtime tumor-tracking radiotherapy system (RTRT). Phys Med. 2016 Feb;32(2):305-11. doi: 10.1016/j.ejmp.2015.10.093.

[25] Michalski D, Sontag M, Li F et al. Four-dimensional computed tomography-based interfractional reproducibility study of lung tumor intrafractional motion. Int J Radiat Oncol Biol Phys. 2008 Jul 1;71(3):714-24. doi: 10.1016/j.jijobp.2007.10.038.

[26] Purdie TG, Moseley DJ, Bissonnette JP et al. Respiration correlated cone-beam computed tomography and 4DCT for evaluating target motion in stereotactic lung radiation therapy. Acta Oncol. 2006;45(7):915-22. doi: 10.1080/02841860600907345

[27] Ruben JD, Seeley A, Panettieri $V$ et al. Variation in lung tumour breathing motion between planning four-dimensional computed tomography and stereotactic ablative radiotherapy delivery and its dosimetric implications: Any role for four-dimensional set-up verification?. Clin Oncol (R Coll Radiol). 2016 Jan;28(1):21-7. doi: 10.1016/j.clon.2015.08.010.

[28] Seregni, M., Pella, A., Riboldi, M., Orecchia, R., Cerveri, P., \& Baroni, G.. Real-time tumor tracking with an artificial neural networks-based method: a feasibility study. Phys Med. 2013 Jan;29(1):48-59. doi: 10.1016/j.ejmp.2011.11.005.

[29] Torshabi, A. E., Pella, A., Riboldi, M., Baroni, G. Targeting accuracy in real-time tumor tracking via external surrogates: a comparative study. Technol Cancer Res Treat. 2010 Dec;9(6):551-62. doi/10.1177/153303461000900603

This article is protected by copyright. All rights reserved. 
Table 1. Distribution of the selected evaluation metrics of the coverage volume histograms (CVH) in the three Lung Optimized treatment (LOT) modalities: C90 and C95 refer to the coverage of the 90\% and $95 \%$ of the clinical target volume (CTV) obtained when clinical safety margins are used. In our Institution, planning target volume (PTV) is defined as end-exhale CTV $+3 \mathrm{~mm}$ in 2-View and as internal target volume (ITV) $+5 \mathrm{~mm}$ in 0-View. In the 1-View, the ITV encompasses only tumor motion along the non-visible direction. Then, non-uniform ITV to PTV margins are applied: $3 \mathrm{~mm}$ and $5 \mathrm{~mm}$ in the visible and non-visible direction, respectively. Percentage of patients satisfying coverage criteria are enlisted.

\begin{tabular}{|c|c|c|c|c|c|c|}
\hline \multirow[b]{2}{*}{$\begin{array}{l}\text { Treatment } \\
\text { modality }\end{array}$} & \multicolumn{3}{|l|}{ C90 } & \multicolumn{3}{|l|}{ C95 } \\
\hline & Median \pm IQR (range) [\%] & $\begin{array}{l}\mathrm{C} 90 \geq 90 \% \\
\text { N pts (\%) }\end{array}$ & $\begin{array}{l}\mathrm{C} 90 \geq 95 \% \\
\text { N pts (\%) }\end{array}$ & Median \pm IQR (range) [\%] & $\begin{array}{l}\mathrm{C} 95 \geq 90 \% \\
\text { N pts (\%) }\end{array}$ & $\begin{array}{l}\text { C95 } \geq 95 \% \\
\text { N pts (\%) }\end{array}$ \\
\hline 2-view & $98.3 \pm 2.4(82.8-99.1)$ & $28(93)$ & $26(87)$ & $97.0 \pm 6.5(77.7-99.1)$ & $27(90)$ & $21(70)$ \\
\hline $\begin{array}{l}\text { 1-view (A- } \\
\text { B) }\end{array}$ & $99.1 \pm 0.6(71.0-99.1)$ & $57(95)$ & $52(87)$ & $98.8 \pm 3.4(68.6-99.1)$ & $53(88)$ & $51(85)$ \\
\hline 0-view & $99.1 \pm 1.0(55.0-99.1)$ & $25(83)$ & $25(83)$ & $99.0 \pm 4.9(52.9-99.1)$ & $25(83)$ & $19(63)$ \\
\hline
\end{tabular}
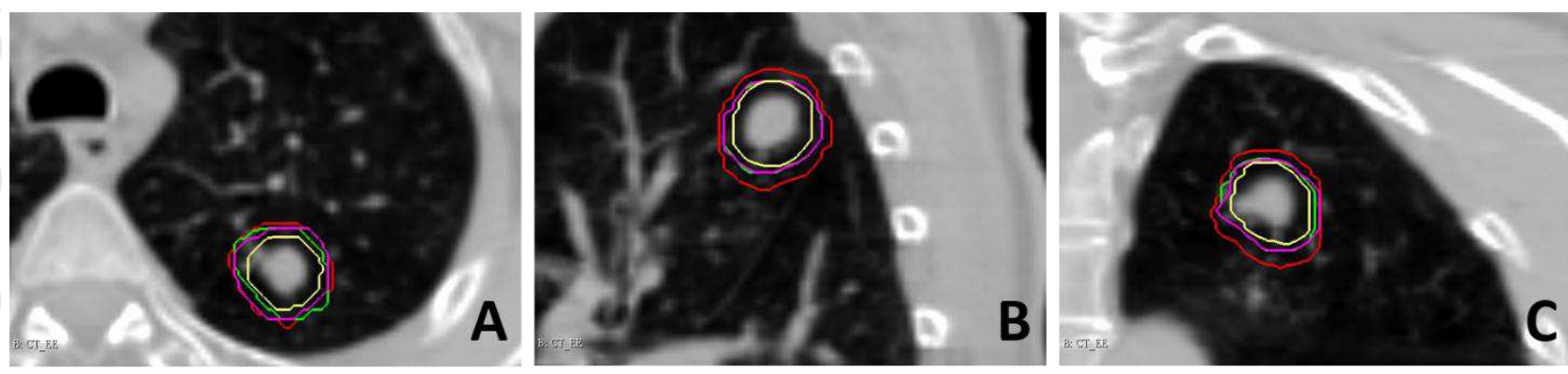

A: CTV/PTV intersection for each image

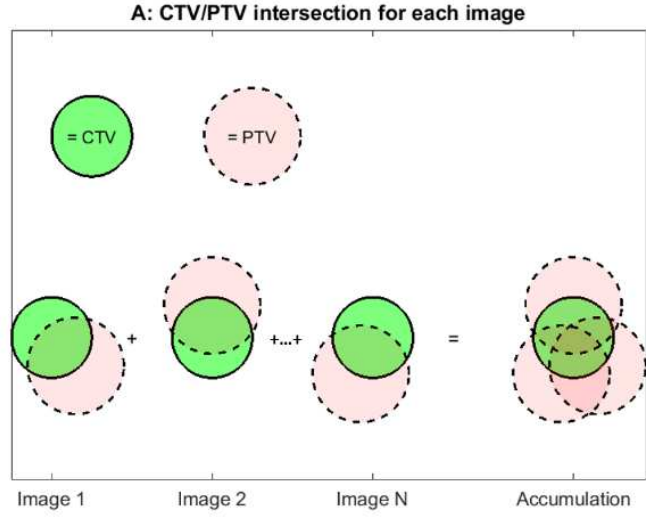

B: CTV coverage [\%]

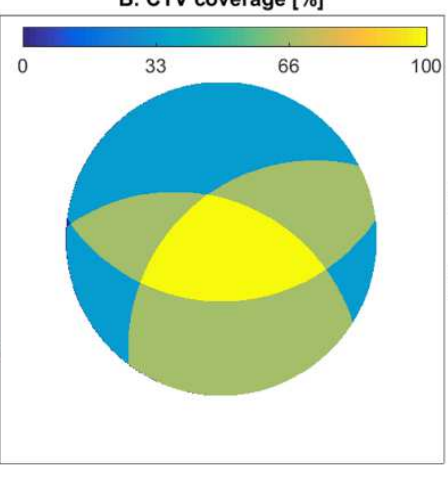

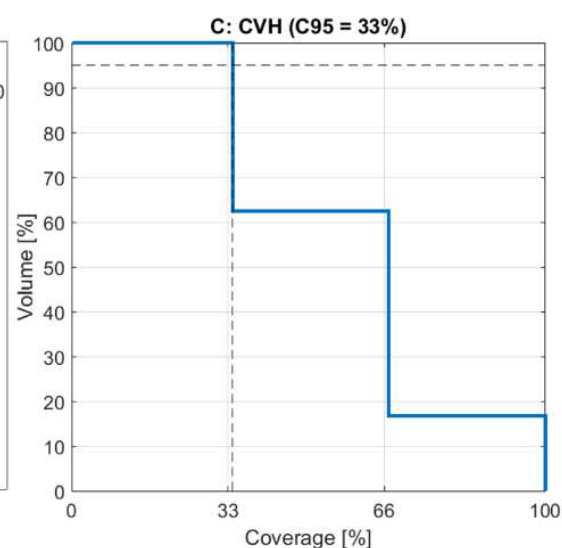

This article is protected by copyright. All rights reserved. 

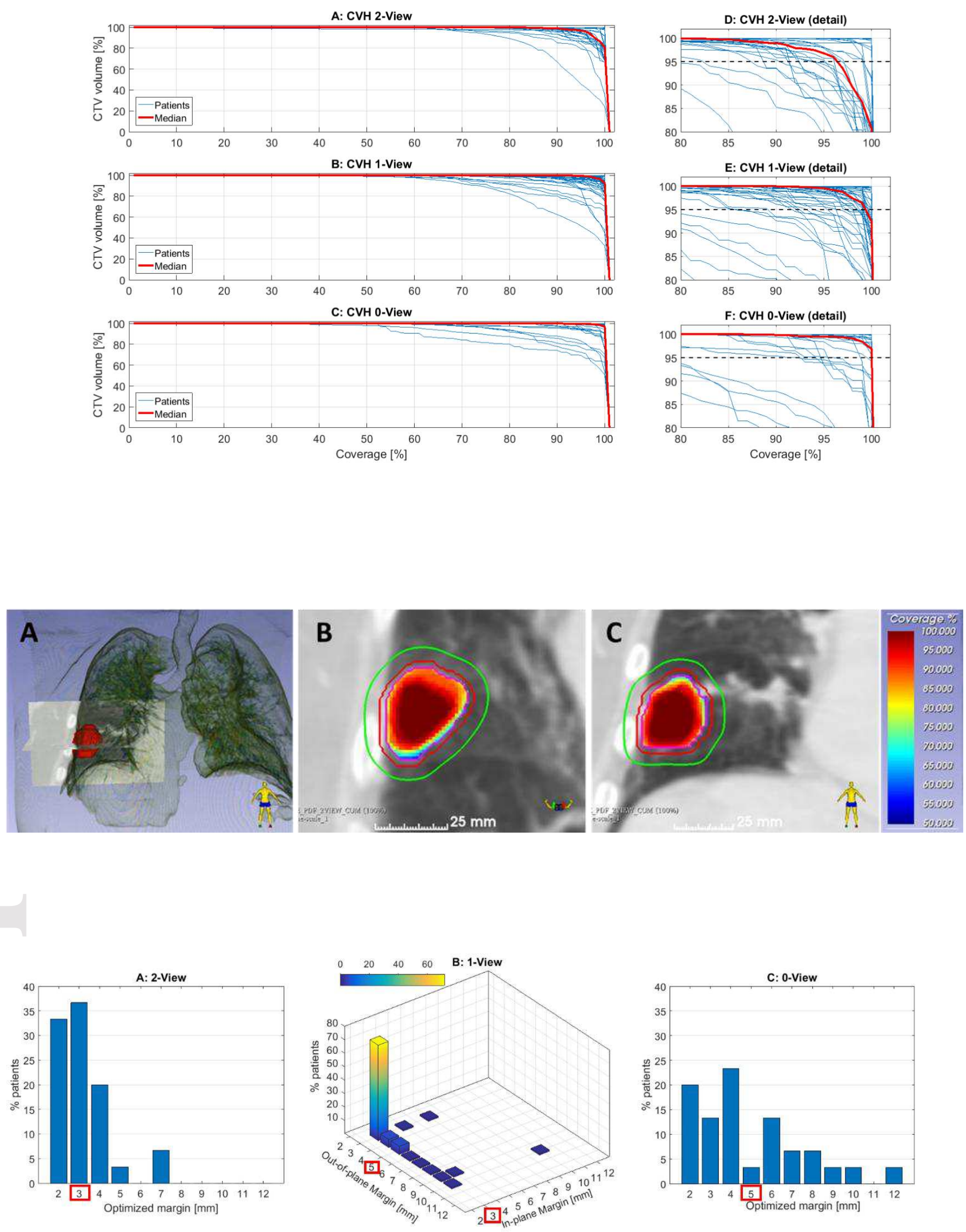

This article is protected by copyright. All rights reserved. 